\title{
Einfluss der Alterung auf das Ermüdungsverhalten
}

\section{von Knochenzementen}

Uwe Soltész ${ }^{1}$, Roland

Schäfer ${ }^{1}$, Klaus-Dieter Kühn²

\section{Schlagworte:}

Knochenzemente -

Alterung - Ermüdung

- Wechsellast-

Verhalten

\begin{abstract}
Knochenzemente zeigen im Allgemeinen ein ausgeprägtes Ermüdungsverhalten. Häufig wirken sich dabei Unterschiede in der Zusammensetzung, der Herstellung oder den Umgebungsbedingungen deutlich stärker auf die Langzeitfestigkeit aus als auf die üblicherweise ermittelte quasistatische Festigkeit. In dieser Studie wird nun als weiterer möglicher Parameter untersucht, ob auch Alterungserscheinungen, denen Polymere oft unterliegen, das Ermüdungsverhalten beeinflussen können. Die Ergebnisse zeigen, dass solche Effekte bei den hier untersuchten Standard-Acrylatzementen vernachlässigbar sind.
\end{abstract}

\section{Einleitung}

Moderne Knochenzemente, wie sie heute für die Einbettung und Fixierung von hoch belasteten Endoprothesen und Implantaten eingesetzt werden, basieren nahezu ausschließlich auf Acrylaten [1]. Unter Wechsellast weisen sie deshalb ein ausgeprägtes Ermüdungsverhalten auf. Üblicherweise vermindern sich ihre Festigkeiten nach praxisrelevanten Lastspielzahlen (d.h. im Bereich bis zu 107 Zyklen) auf nur noch 20 bis $40 \%$ ihres quasistatischen Festigkeitswertes. Während sich jedoch die quasistatischen Ausgangsfestigkeiten der verschiedenen Zemente zumeist nur geringfügig unterscheiden [1], zeigt sich in vielen Fällen, dass das Wechsellastverhalten zusätzlich sehr empfindlich auf verschiedene Parameter der Herstellung, der Zusammensetzung oder der Umgebungsbedingungen reagiert [2]. Deshalb ist die Quantifizierung des Wechsellastverhaltens von großer Bedeutung für die Bewertung der einzelnen Werkstoffe im Hinblick auf den Einsatz in vivo sowie zur Differenzierung des möglichen Einflusses diverser Material parameter.

So wirkt sich natürlich zunächst die Zusammensetzung der auf Acrylaten basierenden, jedoch chemisch unterschiedlich modifizierten Kunststoffe auf das Langzeitverhalten aus [1, 2]. Weiter können aber z. B. die Anmischbedingungen der üblicherweise aus zwei Komponenten zusammengesetzten Werkstoffe - einer flüssigen Monomer-Phase und einem Pulver aus vorpolymerisierten Partikeln - eine große Rolle spielen [3]. Hier sind vor allem die Anmischtemperaturen und die Verfahren unter Vakuum, welche die Porenbildung verringern oder vermeiden sollen, zu erwähnen [3, 4, 5]. Solche Effekte können darüber hinaus durch Beimengungen, wie man sie z.B. zur Erhöhung des Röntgenkontrasts (Zirkondioxid, Bariumsulfat), zur Infektionsbekämpfung durch Fremdkeime (Antibiotika) oder zur Induktion des Knochenanwachsens (Hydroxylapatit) zusetzt, übertroffen werden [3]. Starke Eigenschaftsverschlechterungen können auch durch die Sterilisation [6] oder die Umgebungsbedingungen [7] entstehen.

Einen weiteren wichtigen Aspekt für das Langzeitverhalten,

${ }^{1}$ Fraunhofer-Institut für Werkstoffmechanik, Freiburg i. Br.

2 Heraeus Kulzer GmbH \& Co. KG, Wehrheim/Ts. über den bisher relativ wenig bekannt ist, könnten auch Alterungseinflüsse unter in-vivo-Bedingungen darstellen. Von Polymermaterialien ist bekannt, dass sie häufig einer Degradation durch Alterung unterliegen. Entsprechend könnte auch das Festigkeitsverhalten von Knochenzementen im Einsatz beeinflusst werden. Deshalb wurde in den hier vorgelegten Untersuchungen dieser mögliche Effekt exemplarisch an einigen kommerziellen Knochenzementen untersucht.

Zur Ermittlung des Ermüdungsverhaltens wurden in den vergangenen Jahren standardisierte Vorgehensweisen entwickelt, die sich an die quasistatischen Prüfvorschriften nach ISO/DIN anlehnen [8], sich aber in den Beanspruchungs- und Umgebungsbedingungen mehr den in-vivoVerhältnissen annähern [3].

Sie haben sich bisher in vielen Untersuchungen als aussagekräftige und relativ einfache und kostengünstige Methode bewährt.

\section{Methode}

Als Prüfanordnung wird der 4-Punkt-Biegeversuch nach ISO 5833 mit Probenabmessungen von $75 \times 10 \times 3,3 \mathrm{~mm}^{3}$, einem A uflagerabstand von $60 \mathrm{~mm}$ und einem Druckfinnenabstand von $20 \mathrm{~mm}$ verwendet [8]. Die Prüfung erfolgt bei $37^{\circ} \mathrm{C}$ in Ringer-Lösung. Bis zur Prüfung werden die Proben ebenfalls bei $37^{\circ} \mathrm{C}$ in Ringer-Lösung über mindestens einen Monat gelagert. In dieser Zeit erreichen sie eine Wassersättigung von mindestens $95 \%$, wie sie für den Normalfall unter in-vivo-Bedingungen anzunehmen ist. Außerdem sollten danach alle wesentlichen, die Festigkeit möglicherweise noch beeinflussenden Nachpolymerisationsvorgänge abgeschlossen sein, so dass man mit einigermaßen vergleichbaren Ausgangsbedingungen in den Materialeigenschaften für die sich über einen größeren Zeitraum hinziehenden Wechsellast-M essungen rechnen darf.

Für die Bewertung werden zunächst an jeweils 5 Proben die quasistatischen Festigkeiten ermittelt. Hierzu werden die Proben mit konstantem Lastanstieg von $90 \mathrm{~N} / \mathrm{min} \quad \approx 50$ $\mathrm{MPa} / \mathrm{min}$ ) bis zum Bruch belastet. Der Mittelwert der Festigkeiten wird als Referenzwert für dieses Material festgelegt. 
Die Messungen unter Wechsellast folgen dem WöhlerVerfahren. Die Proben werden einer sinusförmigen zyklischen Belastung von $5 \mathrm{~Hz}$ unterworfen, die praktisch einer Schwellbelastung entspricht (Unterlast $5 \%$ des Referenzwertes bzw. der quasistatischen Festigkeit). Sie werden bis zum Bruch gefahren und die Anzahl der ertragenen Zyklen als die jeweiligen Lebensdauern bestimmt. Bei Überschreiten einer maximalen Zyklenzahl von 10 Millionen Zyklen ohne Bruch wird der Versuch beendet ("Durchläufer"). J eweils 5 bis 6 Proben werden auf unterschiedlichen Lastniveaus (d.h. mit gleicher Oberlast) getestet. Diese Lastniveaus werden im Abstand von $10 \%$ der quasistatischen Festigkeit gewählt. Dadurch ergeben sich im allgemeinen 5 bis 6 Laststufen (zwischen 90 und $30 \%$ der quasistatischen Festigkeit).

Alle Versuche erfolgen lastgeregelt, da physiologische Belastungen nahezu ausschließlich kraftgesteuert ablaufen. Verschiebungsgeregelte Vorgehensweisen würden zu einer Unterschätzung des Versagensrisikos führen [9].

\section{Materialien}

Für fünf kommerzielle Zemente auf Acrylat-Basis werden die Alterungseinflüsse miteinander verglichen. Sie wurden als Beispiele für die typischen Unterschiede in Materialzusammensetzung und -verarbeitung der heute verfügbaren Zemente ausgewählt [1]. Einmal geht es um Modifikationen in der Polymermatrix, dann um Unterschiede in der Verarbeitungsviskosität und schließlich um zugesetzte Antibiotika zur Infektionsverhütung, die während der Verweildauer teilweise herausgelöst werden.

REFOBACIN ${ }^{\circledR}$ PALACOS $® R$ ist ein schnell anquellender und daher hoch-viskoser Standardzement auf Basis eines hydrophilen Methylmethacrylat/M ethacrylat-Copolymers mit Antibiotikum-Beimischung $(0,5 \mathrm{~g}$ Gentamicin-Base auf $40 \mathrm{~g}$ Polymer) [A].

PALACOS $\&$ E-FLOW (identisch mit OSTEOPAL $®$ ) ist ein langsam anquellender und daher niedrig-viskoser Standardzement ebenfalls auf Basis eines Methylmethacrylat/Methacrylat-Copolymers $[B,(A)]$.

OSTEOPAL ${ }^{\circledR} G$ ist die antibiotikumhaltige Variante von OSTEOPAL bzW. PALACOS ${ }^{\circledR} E-F L O W \quad(1,0 \mathrm{~g}$ GentamicinBase auf $40 \mathrm{~g}$ Polymer) 1 [A, (B)].

COPAL ${ }^{\circledR}$ ist vergleichbar mit REFOBACIN( PALACOS ${ }^{\circ R}$, enthält jedoch eine Kombination aus zwei verschiedenen Antibiotika (Gentamicin und Clindamicin) mit einer insgesamt höheren Antibiotika-Konzentration (1,0 g Gentamicin-Base $+1,0 \mathrm{~g}$ Clindamicin- $\mathrm{HCl}$ auf $40 \mathrm{~g}$ Polymer) [A].

SIMPLEX ${ }^{\circledR} P$ ist ein mittel-viskoser Zement auf Basis eines Methylmethacrylat-Styrol-Copolymers, das einen hydrophoben Charakter aufweist und daher hinsichtlich der Auslagerung in Wasser zu einem anderen Verhalten führen könnte als die übrigen Zemente [C].

Hersteller der ersten vier Zemente ist die Firma Heraeus Kulzer. Sie werden von der Firma Merck $[A]$ und zum Teil unter anderem Namen auch von der Firma Schering Plough [B] vertrieben.

Der Hersteller [C] des fünften Zements ist die Firma Stryker Howmedica [1].

Die aus diesen Zementen gefertigten Proben wurden zunächst, wie vorher beschrieben, für mindestens einen

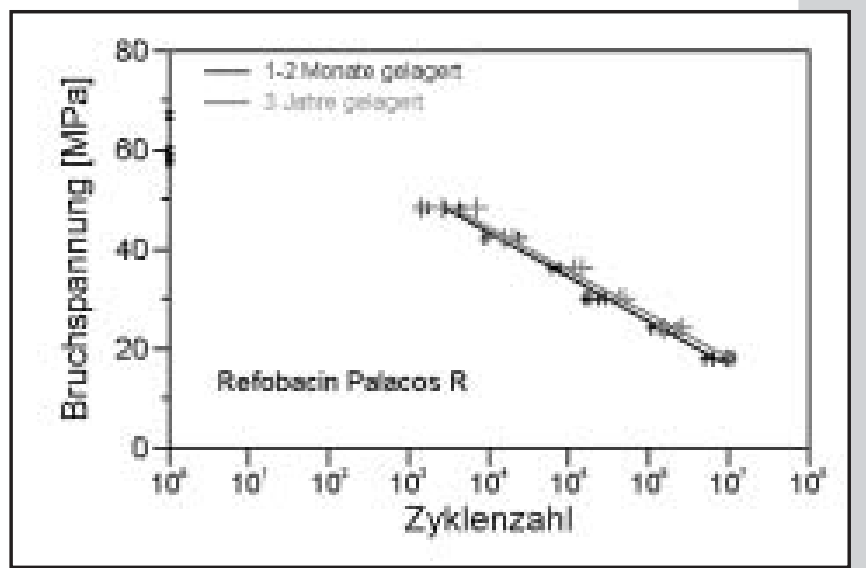

Abb. I: Wöhler-Kurven für REFOBACIN PALACOS R nach $\geq$ I Monat bzw. 3 Jahren Alterung

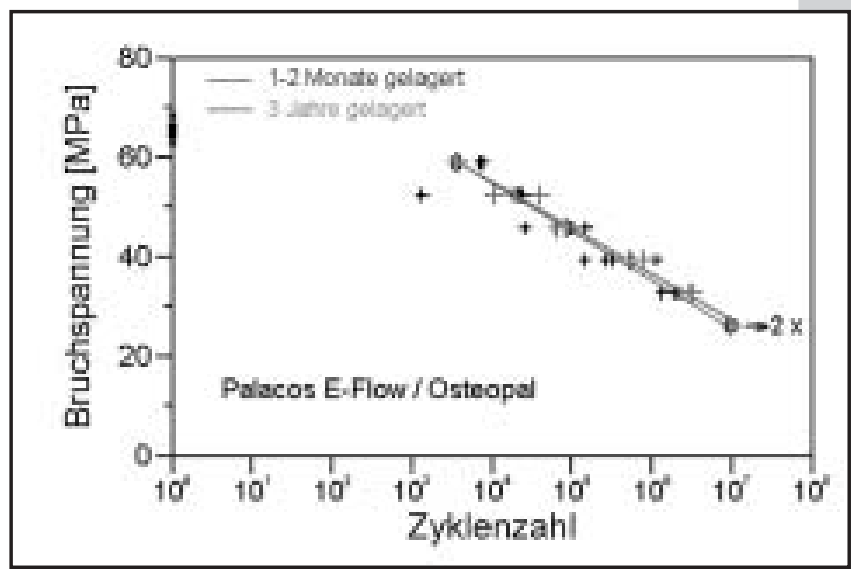

Abb. 2: Wöhler-Kurven für PALACOS E-FLOW (OSTEOPAL) nach $\geq$ I Monat bzw. 3 Jahren Alterung

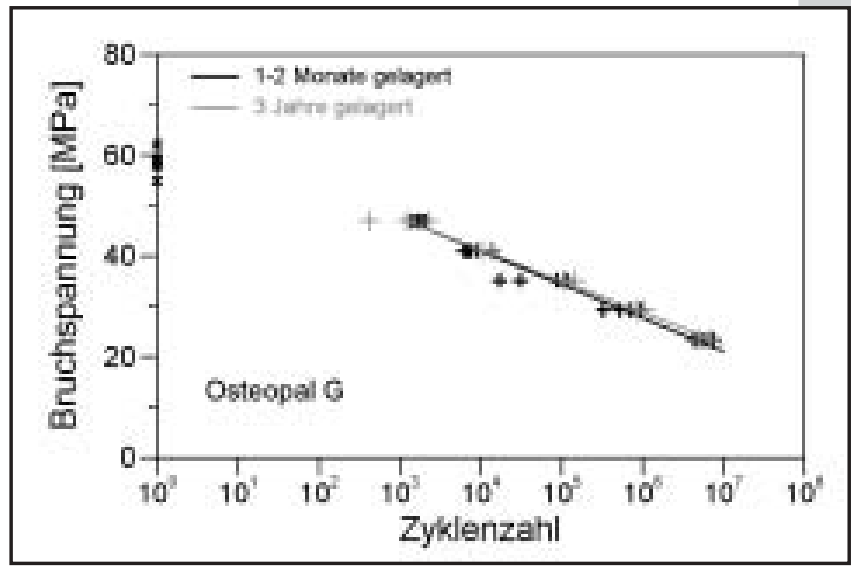

Abb. 3: Wöhler-Kurven für OSTEOPAL G (REFOBACIN PALACOS E-FLOW) nach $\geq$ I Monat bzw 3 Jahren Alterung

Monat bis zur weitgehenden Wassersättigung in RingerLösung bei $37^{\circ} \mathrm{C}$ gelagert. Nach dieser Lagerung wurde ein Teil der Proben in der angegebenen Weise quasistatisch bzw. unter Wechsellast getestet. Die restlichen Proben wurden bei Raumtemperatur weiter in Wasser gelagert. Von diesen wurden die ersten drei der oben aufgeführten Materialien etwa drei Jahre und die beiden letzten etwa ein Jahr nach Herstellung getestet.

Die Daten für die hier dargestellten Zemente wurden zum 


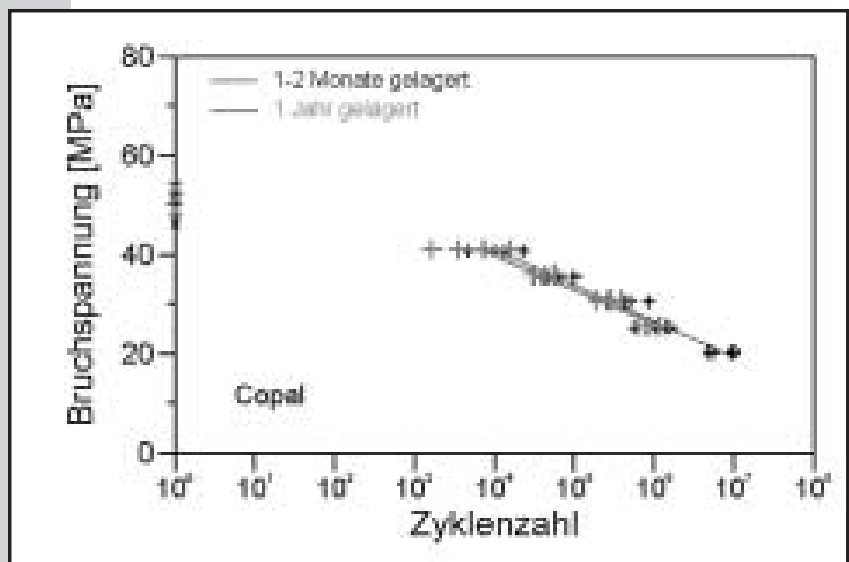

Abb. 4: Wöhler-Kurven für COPAL nach $\geq$ I Monat bzw. I Jahr Alterung

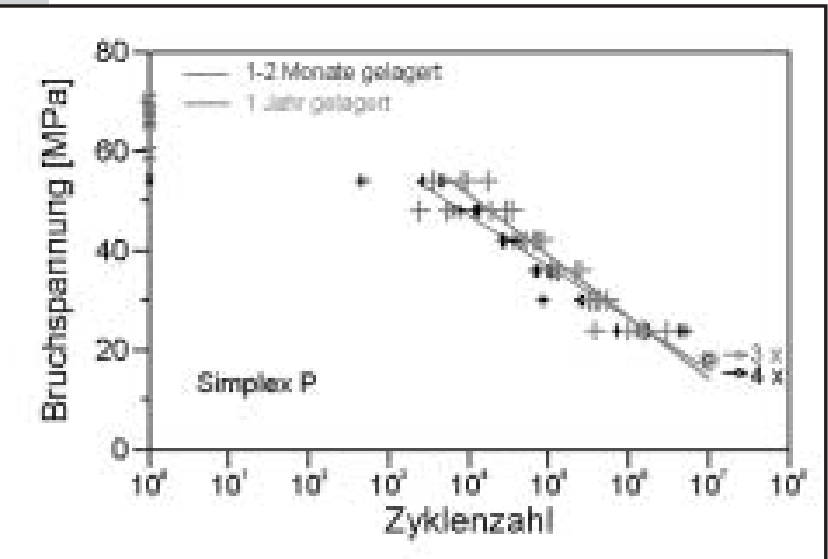

Abb. 5: Wöhler-Kurven für SIMPLEX P nach $\geq$ I Monat bzw. 3 Jahren Alterung

Teil schon früher in verkürzter Form präsentiert [10, 11], für den Vergleich hier jedoch ergänzt und neu aufbereitet. Dies bezieht sich vor allem auf die Vorgehensweise bei der linearen Regression mit der Lebensdauer als abhängiger Variablen und einer vergleichbaren Bewertung der Durchläufer. Diese blieben unberücksichtigt, wenn sie zu weit unterhalb der Regressionsgeraden lagen und die Kurve unzulässig nach unten gezogen hätten.

\section{Ergebnisse}

Die Ergebnisse sind in Form von Wöhler-Diagrammen in den Abbildungen 1 bis 5 in vollständiger Form wiedergegeben.

Auf der Ordinate sind jeweils die quasistatischen Werte $(x)$ aufgetragen, Kreuze (+) stellen die unter Wechsellast gebrochenen Proben dar, Kreise (0) die Durchläufer. Die Einzelmessungen werden durch lineare Regression in der gewählten halblogarithmischen Darstellung angepasst, wobei sowohl die unter Wechsellast gebrochenen Proben $(+)$ als auch die Durchläufer (0) berücksichtigt werden und die Lebensdauer auf der logarithmischen Zeitachse als die abhängige Variable angesehen wird. Es werden jeweils die kurz gelagerten Proben (1-2 Monate) mit den gealterten verglichen. Sie sind durch unterschiedliche Graustufen gekennzeichnet.

In Tabelle 1 werden zusätzlich auch einzelne Festigkeitskennwerte miteinander verglichen, und zwar die jeweiligen quasistatischen Ausgangswerte mit den Werten, die sich für 10 Millionen Zyklen aus den Ausgleichsgeraden ergeben.

\section{Schlussfolgerung}

Die Ergebnisse führen zu einem weitgehend einheitlichen Bild. Die Wöhler-Geraden für die miteinander verglichenen Lagerzeiten unterscheiden sich innerhalb der Streubreite nicht, d.h sie sind praktisch identisch. Lediglich bei SIMPLEX $P$ deuten sich möglicherweise unterschiedliche Steigungen an, die bei einer Extrapolation zu noch größeren Lastspielzahlen eine Festigkeitsminderung durch Alterung ergeben würden. Es ist jedoch unklar, ob in diesem Zeitbereich eine Beschreibung der Wöhler-Kurve durch eine Gerade überhaupt noch zulässig ist. Die in diesem Diagramm eingezeichneten 3 bzw. 4 Durchläufer könnten jedenfalls auf ein Abknicken der Kurven hindeuten.

Zusammenfassend lässt sich feststellen, dass Alterungseinflüsse bei den hier untersuchten, exemplarisch ausgewählten Zementen vernachlässigbar sind und weder durch Modifikationen des Basismaterials noch der Anfangsviskosität beeinflusst werden. Auch die Zusätze von Antibiotika oder deren teilweise Herauslösung während der Lagerzeit bewirken offensichtlich bei

\begin{tabular}{|lccc|}
\hline Material & Lagerzeit & $\begin{array}{c}\text { Festigkeit } \\
\text { quasistatisch } \\
{[\mathrm{MPa}]}\end{array}$ & $\begin{array}{c}\text { Festigkeit } \\
10^{7} \text { Zyklen } \\
{[\mathrm{MPa}]}\end{array}$ \\
\hline Refobacin Palacos A & $\begin{array}{c}\text { 1-2 Monate } \\
\text { 3 Jahre }\end{array}$ & 60,4 & $\begin{array}{c}16,7 \\
18,5\end{array}$ \\
\hline Palacos E-Flow & $\begin{array}{c}\text { 1-2 Monate } \\
\text { 3 Jahre }\end{array}$ & 65,6 & 25,7 \\
& 1-2 Monate & 58,6 & 27,2 \\
\hline Osteopal G & 3 Jahre & - & 22,0 \\
& 1-2 Monate & 50,9 & 19,3 \\
\hline Copal & 1 Jahr & 51,5 & $(19,4)^{*}$ \\
\hline Simplex P & 1-2 Monate & 64,5 & 15,9 \\
& 1 Jahr & 66,3 & 14,9 \\
*) extrapolierter Wert, Messung nur bis $10^{6}$ Zyklen & \\
\hline
\end{tabular}

Tab. I: Vergleich der quasistatischen Festigkeiten mit den Ermüdungswerten nach 10 Millionen Zyklen für die untersuchten Knochenzemente diesen Materialien keine Veränderung der Wechsellastfestigkeit. Dieser Befund lässt sich wahrscheinlich auch auf die meisten heute verwendeten Knochenzemente übertragen, da diese nahezu ausschließlich auf Acrylaten basieren und Eigenschaftsänderungen durch vergleichbare Modifikationen in der Zusammensetzung erreicht werden.

\section{Abstract}

Bone cements normally exhibit a distinct fatigue behaviour. Frequently differences in the composition, the preparation or the environmental conditions additionally influence the long-term strength. The changes by fatigue are mostly more pronounced than those of the usually determined quasistatic strength. In this study as a further possible influence it is investigated whether ageing effects to which polymers are often subject, may change the fatigue 


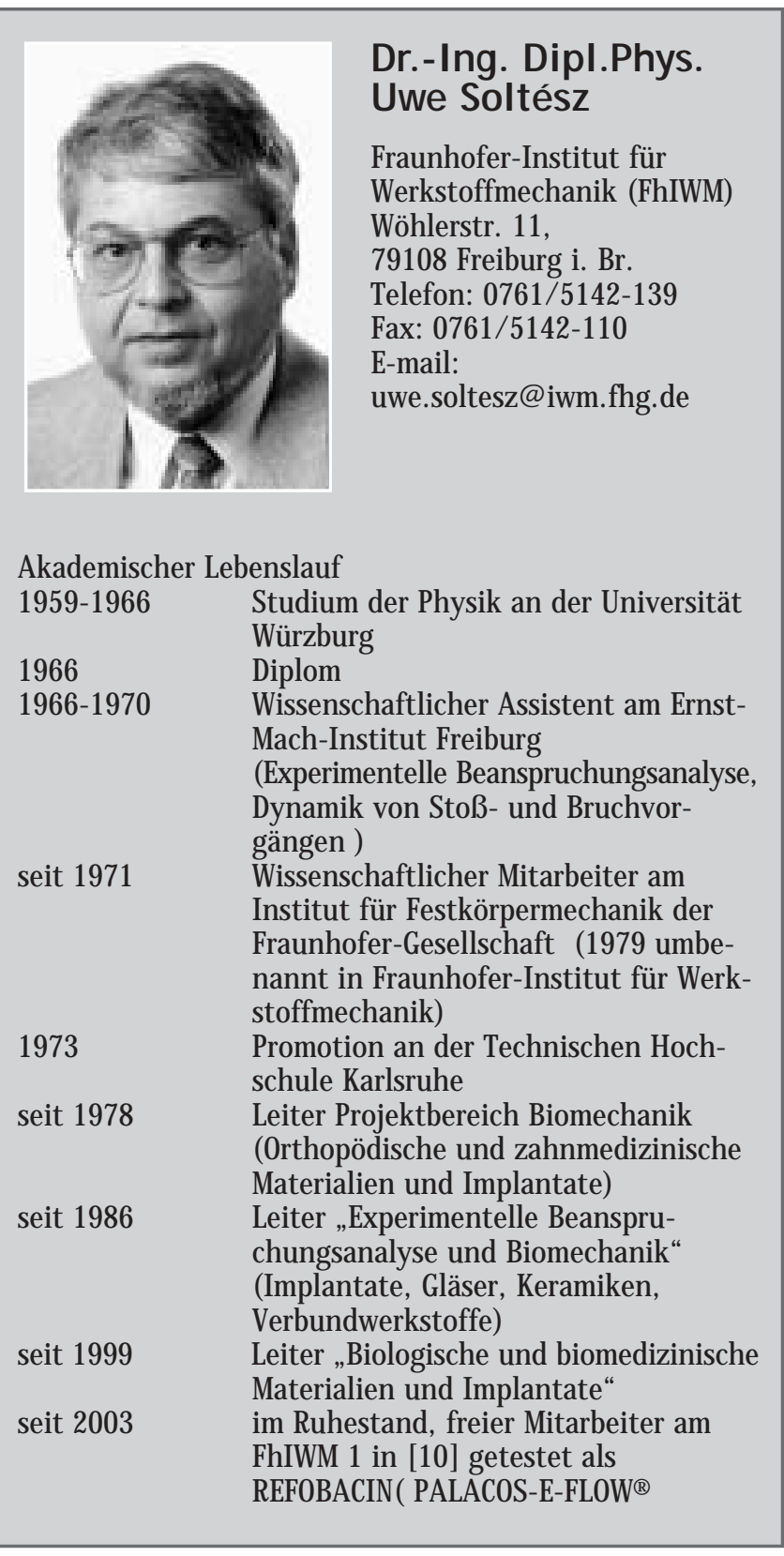

behaviour, too. The results show that for the standard acrylate-based cements considered here, such influences can be neglected.

\section{Literatur}

[1] Kühn K.-D.: Knochenzemente für die Endoprothetik. Ein aktueller Vergleich der physikalischen und chemischen Eigenschaften handelsüblicher PM M A-Zemente. Springer-Verlag, Heidelberg 2001

[2] Soltész U., Schäfer R., Kühn K.-D.: Comparison of the fatigue behaviour of commercial bone cements. Transactions of the Sixth World Biomaterials Congress, Kamuela/Hawaii 2000, Vol III, 1458

[3] Soltész U., Schäfer R.; Kühn K.-D.: Einfluss von Anmischbedingungen und Beimengungen auf das Ermüdungsverhalten von Knochenzementen. Berichtsband 1. Tagung DVM-AK Biowerkstoffe: "Mechanische Eigenschaften von Implantatwerkstoffen“, Berlin 1998, 89-94
[4] Soltész U., Ege W.: Influence of mixing conditions on the fatigue behaviour of an acrylic bone cement. Book of Abstracts, 10th Europ. Conf. on Biomaterials, Davos 1993, 138

[5] Tepic S., Soltész U.: Fatigue strength of bone cement with simulated stem interface porosity. 14th Europ. Conf. on Biomaterials, Den Haag 1998

[6] Soltész U., Tepic S.: Einfluss der Sterilisation auf das Ermüdungsverhalten von Knochenzementen. Werkstoffwoche 1998, Band IV: Werkstoffe für die Medizintechnik, WileyVCH, Weinheim 1999, 239-243

[7] Schäfer R., Soltész U., Kühn K.-D.: Influence of environmental conditions on the fatigue strength of bone cements. 17th Europ. Conf. on Biomaterials, Barcelona 2002

[8] ISO 5833: Implants for surgery - Acrylic resin cements. ISO 1992.

[9] Soltész U.: The influence of loading conditions on the life-times in fatigue testing of bone cements. J. Mater. Sci.: Materials in Medicine, 5 (1994), 654-656

[10] Soltész U., Ege W.: Influence of ageing on the fatigue behaviour of three acrylic bone cements. Transactions 1st Ann. Conf. of the Europ. Orthopaedic Research Society, Paris 1991, 80

[11] Soltész U., Schäfer R., Kühn K.-D., Ege W.: Fatigue behaviour after ageing of a bone cement with high content of antibiotics. Proc. ESB'99 (15th Europ. Conf. on Biomaterials), Bordeaux 1999 